\title{
Response of the Newborn When Gently Accosted by the Mother Immediately after Birth and Subsequent Growth and Development
}

\author{
Hiroshi Nakajima \\ Nakajima Maternity Hospital, Saitama, Japan
}

(Received for publication on June 14, 1994)

\begin{abstract}
Observations were made on the emotion and behavior of the newborn placed beside the mother immediately after vaginal delivery. The newborn after listening the mother's gentle voice will soon show a peaceful Buddha-like facial appearance and cease to cry. The newborn will not have opened it's eyes for more than 10 minutes unless bathed after birth and placed beside the mother and will hardly move either extremity. After about 10 minutes, bilateral movement of the fingers, wrists and forearms was observed. This newborn will not cry at all for several hours thereafter. On the other hand the infant born to a heavy smoker would not cease to cry and would continue to maintain an appearance of anxiety even when gently accosted by the mother. The newborn about $\mathbf{1 0}$ minutes after birth has the ability to search for the mother's nipple. (Keio J Med 43 (3): 167-170, September 1994)
\end{abstract}

Key words: delivery, newborn, emotion, behavior

\section{Mothers and the Newborn of the Present Study}

The subjects of the present study were various cases of mothers and their babies born at the Nakajima Maternity Clinic by vaginal delivery from 1 April to 31 December 1993.

\section{Observation Conditions}

Management of the mother and the newborn immediately after delivery

(1) Immediately after vaginal delivery, the amniotic fluid was aspirated and the amniotic fluid and blood of the newborn were removed by absorbent paper. The midwife was made to handle the newborn smoothly and gently. (2) Next, the infant was placed on the right side of the mother and then draped with underwear. Immediately thereafter, the mother was asked to gently accost the newborn. The time required to this step was 4 to 5 minutes. (3) This condition was continued for $20-30$ minutes to 3 hours. The mother was asked not to embrace or touch the newborn. When the father was present at the time of delivery, he was requested not to accost the newborn. (4) After inducing breast searching motion on the part of the infant as described later, the infant was made to search for the mother's nipple. (5) After 20-30 minutes to 3 or 4 hours, the infant was bathed for the first time. (6) Until the newborn was bathed, the mother was kept in the delivery bed. (7) It was only after the newborn was bathed that the mother was returned to the ward and the majority of the infants were placed in the same room as their mothers. (8) From ten at night to nine in the morning, the mother and infant were separated with the nurse made responsible for the management of the infants during the night. (9) From the day after delivery, the mother and infant shared the same room except during the night.

\section{Method of observation}

Video recording by VHS video camera was made of

中島 洋

Reprint requests to: Dr Hiroshi Nakajima, Nakajima Maternity Hospital, 2-10-5 Shimotoda, Toda, Saitama 335, Japan 
the behavior and facial appearance of the infant continuously for 20 minutes from the time of delivery and briefly from 6 to 7 times thereafter for about three hours.

\section{Results}

\section{Observation of the newborn within several hours after delivery}

Typical cases of infants gently accosted by their mothers: (1) The newborn having an Apgar score of 10 points immediately cried after delivery and the cry of almost all of the infants showed a completely identical pattern, a cry which can be considered to be unpleasant. Most of the infants cried with their eyes closed. (2) When the mother gently accosted the crying infant, the infant ceased to cry within several seconds to $20-30$ seconds and the facial appearance changed to a Buddha-like countenance (facial appearance of relief). (3) After (2), the infant no longer cried at all and appeared to be listening intently to the mother's voice with the eyes closed. (4) During (3), most of the infants hardly showed any movement of the upper and lower extremities nor was there any appreciable movement of the head or lips. However, it was tension which was observed in the muscle and not paralysis. (5) After about 10 minutes, bilateral movement of the fingers, wrists and forearms observed. (6) The movement of the hands consisted of extension of the fingers together with movement of the fingers as if in search of something. Then movement of

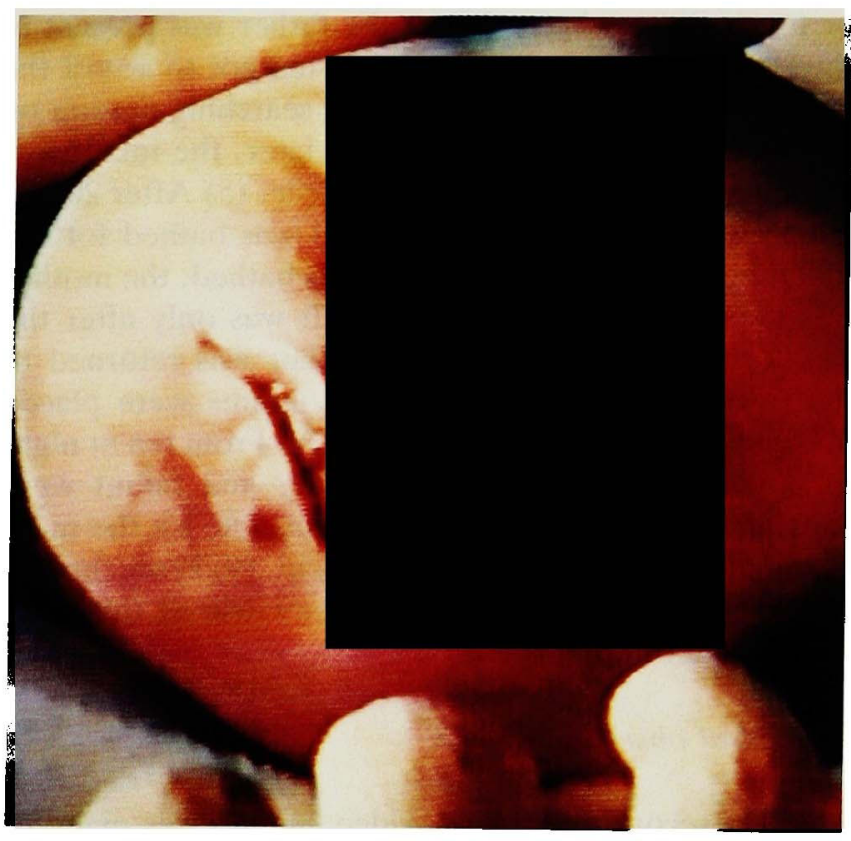

Fig 1 The tranquillity of Buddha shows in the newborn's face right after birth if it hears mother's voice. the lips commenced. This movement and that of the head appeared to be movements in search of something. The eyes were either closed or opened during these movements. (7) In the cases of most babies, both eyes did not open simultaneously. One eye was opened first and the other eye was opened after 2 or 3 minutes. (8) The cry of most of the newborn who cried about 10 minutes after delivery was small and was not the typical cry at delivery. (Utterance was made with a facial appearance unlike that at time of cry at delivery.) (9) When the mother's nipple was brought within $4-5 \mathrm{~cm}$ of the infant's lips 10 or 20 minutes after delivery, after the infant showed these movements of the lips, head and hands, the infant soon commenced a movement in search for the nipple. Just before and after this movement, the infant showed radiant facial appearance as if the infant had confirmed the presence of the nipple and upon locating the nipple the facial appearance changed to one of satisfaction. (The mother did not provide any assistance in the infant's search for the nipple. However, before the search for the nipple, the mother assumed a position of placing the breast against the infant's cheek and nose to facilitate the movement of the infant to locate the nipple. The infant would extend the lower extremities and use the upper extremities in search for the nipple and would finally move the head to reach the nipple, but could not move the upper part of the body with the upper extremities. The infant could move the entire body forward with the use of the lower extremities but not backward.) (10) Upon locating the nipple, some of the infants would suck for several minutes and release the nipple with a facial appearance of relief, while other infants would continue to suck and would cry in anger when the nipple was removed from the mouth. About two-thirds of the infants could locate the nipple. (11) When the mother gently accosted the infants lying adjacent to the mother, most of the infants, whether able to locate the nipple or not, appeared to be sleeping without crying at all. During this period, there was no ocular movement not was there hardly any movement of both extremities and lips. The mothers used the expression that "they appeared to be dead", but when gently accosted by the mother, they responded with a smiling facial appearance with a countenance of relief.

Atypical cases after being gently accosted by the mother $\{$ After (1) of $A\}$ : The infant born to a heavy smoker would not cease to cry and would continue to maintain an appearance of anxiety even when gently accosted by the mother.

The infant who were first bathed without being gently accosted by the mother: When a normally delivered newborn having an Apgar score of 10 points was bathed without being gently accosted immediately after birth or 
embraced by the mother and left alone after being bathed, the infant assumed a facial appearance of anxiety. The facial appearance of anxiety did not dissipate when first embraced and gently accosted by the mother 15 minutes after birth and it required 20 minutes before the infant could assume a facial appearance of relief. Thereafter this infant even when left alone continued to show a calm facial appearance without a facial appearance of anxiety.

The subsequent course of infants gently accosted by the mother immediately after birth

Infants during the neonatal period (1-6 days after birth: (1) The infants always assumed a calm facial appearance. (2) The infants hardly ever became fretful or cried. It was only when they wanted to be fed or when the diapers were wet that they would cry.

Infants 1 month after birth: (1) The infants hardly ever cried at night and did not cry except when they wanted milk or when the diapers were wet. (2) The infants rarely cried and were so calm and quiet that the family members would become worried.

\section{Consciousness of the mothers}

Consciousness of the mothers immediately after delivery: After 10 minutes following delivery, when the mother began to note that the infant opened its eyes, moved its hands and mouth, and changed its facial appearance, a change of the mother's consciousness toward her offspring commenced.

Consciousness of mothers in confinement (1-6 days): A multipara would invariably realize that the infant was quiet, did not cry as much as her carlier child and had a more richly expressive facial appearance.

Consciousness of mothers after one month of confinement: It was the impression of primipara that childcare was easier than expected. a: The infant did not cry at night. $b$ : The infant did not cry without a reason. $c$ : The infant cried only when milk was wanted or when the diapers had to be changed.

\section{Discussion}

For many years the author has made a video recording of the newborn at time of delivery and observed that the facial appearance of the newborn changed according the management pattern of the newborn immediately after delivery. Unlike the heretofore employed pattern of management, the author, therefore, has placed the newborn immediately after delivery under a natural condition, that is, placed the newborn adjacent to the mother prior to bathing, and has requested the mother to gently accost the newborn in an effort to observe how the facial appearance of the newborn would change. Furthermore, consideration was given to what the movement, behavior, and facial appearance of the newborn under natural condition would imply.,

Obstetricians have employed the cry of the newborn at time of delivery as a barometer of health of the newborn. This is because this cry implies adequate pulmonary respiration. Is this cry only an utterance accompanying adequate pulmonary respiration or does it imply anything else?

The author has observed that the newborn becomes calm, assumes a Buddha-like facial appearance, and ceases to cry immediately after listening to the mother's gentle voice. The mother's gentle voice causes the infant's cry to cease and the facial appearance of the infant to become calm. Does the newborn feel that it is not necessary to cry if mental calmness is reached? If so, it may be speculated that the act of crying is a means of emotional expression of anxiety, self-assertion and the like.

Why does the infant cease to cry immediately after birth upon listening to the mother's voice and why does the infant continue to cry if not gently accosted by the mother? Does the infant identify its own mother from the voice of the mother which it heard while in the uterus and then become calm upon the confirmation of her presence? Does the newborn possess such a mechanism while in the uterus (for example, synthesis or secretion of intracranial substances such as opium-like substances)? Is it a mechanism obtained by delivery $?^{1,2}$

Following the cry immediately after delivery and active movement of the both extremities for several minutes, the newborn ceases active movement for more than ten minutes with the eyes closed. What does this imply?

Does the central nervous system having a mechanism similar to that in the uterus function at the same level as in the uterus?

The newborn immediately after birth actively moves both extremities for several minutes and thereafter for a given period the movement of both extremities becomes weak with both eyes closed, but 10-20 minutes later the movement of the upper extremities, lips, and head is resumed with the eyes opened.

Does this imply that immediately after delivery there is, accompanying delivery, the presence of a mechanism of the central nervous system unlike that in the uterus or does it indicate that the level of this mechanism changes following delivery?

What are the factors which influence these? What are involved in the cry of the newborn at time of delivery other than internal changes of the fetus due to mechanism of delivery, stimulus due to changes in external environ- 
ment, and respiratory and circulatory changes in the new physiological functions following birth?

We can think that the series of processes such as process of crying at time of birth and the function of hearing one's own crying at time of birth are absent in the uterus. Cannot this be considered a factor which may influence the foregoing mechanism?

Next, the relationship between crying at time of birth and behavior of the newborn immediately after birth will be considered. The newborn immediately after birth can hear the voice of the mother and express a facial appearance of relief and about 10 minutes later the movement of the hands and lips in search for the mother's nipple begins together with the opening of the eyes. Furthermore, the newborn, while continuing to cry and in a state of anxiety, commences about 10 minutes later a movement in search for the mother's nipple is an instinctive function and is different from the emotion of the newborn.

The newborn who has become calm after listening to the mother's voice immediately after birth will continue to possess stable, composed emotions. Such a newborn will, without the provision of any special childcare, not cry at all at night and will smile while sleeping. Most of the infants are calm and peaceful and will not cry even when blood is drawn for examination. Though the mother gently accosted the fetus during pregnancy, the newborn will not become a calm and peaceful infant unless the mother gently accosted the newborn immediately after birth. Furthermore, if the newborn was bathed immediately after birth and showed a facial appearance of anxiety before meeting the mother, the newborn did not immediately show a facial appearance of composure though the mother gently accosted the newborn more than 10 minutes after birth.

The foregoing suggests that the time of imput of the mother's voice has a decisive implication. With regard to the input of the mother's voice, this indicates that the brain activity of the newborn immediately after birth is not the same as that of the fetus in utero or that of the newborn 20-30 minutes after birth.

As for communication between the mother and the infant, it is important that the newborn immediately following vaginal delivery be placed in a natural condition beside the mother and that the mother gently accost the newborn. This is considered to be a decisive condition in bringing about emotional stability of the newborn.

These newborn cry very little and sleep well. These imply that their energy metabolism is low. On the other hand, their mothers begin to produce adequate milk 4 or 5 days after delivery. These are considered to be phenomena physiologically beneficial to both the mother and the newborn.

It is the sincere hope of the author that this observation of his would be useful in the study of communication between the mother and the infant from the standpoint of both the infant and the physiology involved.

\section{Conclusion}

It is the communication between the mother and newborn through the gentle verbal communication of the mother to the newborn immediately after birth which gives composure and stability to the emotions of the newborn.

Acknowledgments: This paper was prcsented April 9 at the MotherInfant Interaction workshop of the World Association for Infant Mental Health Regional Meeting Tokyo, 1994. At the workshop, Professor Hannus Papousek, Ludwig Maximillian University, commended my use of "Budda face". I appreciated his understanding and encouragement. The author thanks Hiroshi Nakahama (Emeritus professor, Tohoku University School of Medicine) for his advice and Kenneth Wybrow (Barrister, Sydney, Australia) for this translation.

\section{References}

1. De Casper AJ, Fifer WP: Of human bonding: newborns prefer their mothers' voices. Science 1980, 208: 1174-1176

2. Oshima K: Memory of the fetus. Gendai Shiso 1991, 19: 72-86 (in Japanese)

3. Valman HB, Person JF: What the fetus feels. Br Med 1980, 280: 233-234

4. Von Raffler-Engel W, ed: The Perception by the Unborn, Tokyo, Taishukan Shoten, 1993: 122-124 (in Japanese) 